\title{
Exploración de las relaciones entre clima escolar, satisfacción con la vida y empatía en adolescentes costarricenses
}

\author{
Alvarado Calderón, Kathia \\ Exploración de las relaciones entre clima escolar, satisfacción con la vida y empatía en adolescentes \\ costarricenses \\ Revista Educación, vol. 46, núm. 1, 2022 \\ Universidad de Costa Rica, Costa Rica \\ Disponible en: https://www.redalyc.org/articulo.oa?id=44068165015 \\ DOI: https://doi.org/10.15517/revedu.v46i1.45127
}

\section{(c) (1) $\Theta \Theta$}

Esta obra está bajo una Licencia Creative Commons Atribución-NoComercial-SinDerivar 3.0 Internacional. 


\title{
Exploración de las relaciones entre clima escolar, satisfacción con la vida y empatía en
} adolescentes costarricenses

\author{
Exploring the Relationship of School Climate, Life Satisfaction and Empathy in Costa Rican Adolescents \\ Katbia Alvarado Calderón \\ Universidad de Costa Rica, Costa Rica \\ kathia.alvarado@ucr.ac.cr \\ DOI: https://doi.org/10.15517/revedu.v46i1.45127 \\ Redalyc: https://www.redalyc.org/articulo.oa? \\ iD https://orcid.org/0000-0002-4779-7892 \\ Recepción: 22 Diciembre 2020 \\ Aprobación: 04 Marzo 2021
}

\section{Resumen:}

Este estudio tuvo como objetivo explorar la relación entre la satisfacción con la vida, la respuesta prosocial empática y la percepción del clima escolar, definido este último a partir de cinco dimensiones. En el estudio participaron 401 jóvenes adolescentes entre los 15 y 17 años, pertenecientes a centros educativos públicos de educación diversificada. La investigación fue de tipo correlacional a partir de la utilización de tres instrumentos auto-administrados: el SLSS de Huebner, el Cuestionario de clima escolar de Aron y Milicic y el índice de reactividad interpersonal (IRI). Los análisis estadísticos no paramétricos muestran que no existen diferencias significativas por edad en ninguna de las dimensiones estudiadas, pero sí se encontraron diferencias significativas por género en el índice de reactividad interpersonal. Las dimensiones: respuesta empática, satisfacción con la vida y percepción del clima escolar presentaron asociaciones positivas y significativas entre sí. La dimensión de bullying no se asocia con ninguna de las dimensiones estudiadas. Los instrumentos presentaron buenos valores de confiabilidad. Se concluye sobre la importancia de continuar estudiando los climas sociales escolares en relación con la respuesta empática y la satisfacción con la vida para propiciar ambientes educativos saludables. El papel del personal docente es de gran importancia al respecto de la valoración positiva del clima escolar, así como las condiciones de infraestructura y las actividades extracurriculares que la institución educativa puede ofrecer a la comunidad estudiantil. Se recomienda que programas en comportamiento prosocial en contextos educativos consideren la socialización de género que incide en la manifestación de una genuina preocupación por el dolor de la otredad, este como componente afectivo de la respuesta empática asociado, con mayor frecuencia, a características femeninas.

Palabras Clave: Clima escolar, Satisfacción con la vida, Empatía, Adolescentes.

\section{Abstract:}

An assessment of the relationship existing among life satisfaction, empathic response and school climate, based on a perception of five aspects, was conducted among 401 student participants between the ages of 15-17. This was a correlational study based on the use of three self-administered questionnaires: Huebner's SLSS (Student Life Satisfaction Scale), the Aron and Milicic School Climate Questionnaire and the Interpersonal Reactivity Index (IRI). Non-parametric statistical analysis revealed no significant differences according to age in any of the areas studied. Significant gender differences were observed in the Interpersonal Reactivity Index. Empathic response, satisfaction with life and perception of school climate were associated positively. Bullying was not associated with any of the aspects of the study. All of the questionnaires had good reliability values. It concludes on the importance of continuing to study school social climates in relation to the empathic response and satisfaction with life to promote healthy environments. Teachers play a relevant role in assessing satisfactory school climates, infrastructure conditions and extracurricular activities available to the student community. More programs to promote social skills in educational contexts are necessary for gender mainstreaming and foster genuine concern for the pain of the other, an affective response that shows empathy and is frequently associated with female traits.

KEYWORDS: School Climate, Life Satisfaction, Adolescents, Empathy.

\section{INTRODUCCIÓN}

Desde los años ochenta, la noción de clima escolar, heredera de los estudios de clima organizacional, se ha revisado en diversas investigaciones que han generado una gran cantidad de definiciones e instrumentos 
de investigación. Así, López et al. (2014) incluyen en su descripción de clima escolar "las percepciones, pensamientos y valores que los miembros de un establecimiento educacional construyen de este y de las relaciones que en él se dan" (p. 17); para Turner et al.(2014), el clima escolar se ha comprendido como la conexión psicológica que la persona hace con la institución educativa basada en dos aspectos: el apoyo académico percibido, que se encuentra relacionado con el interés de las personas docentes por ayudar a sus estudiantes así como las políticas institucionales, y el apoyo del grupo social.

Por su parte Berg y Aber (2015) consideran otros aspectos en la definición de los climas sociales en la institución escolar, tales como el apoyo social, la percepción de orden y de justicia, aspectos que permiten interacciones de alta calidad, donde la comunidad educativa se siente segura, respetada y motivada; mientras que para Aron y Milicic (2013) estas percepciones, que constituyen el clima escolar, se refieren a las normas y creencias que lo caracterizan y que se relacionan con diversas dimensiones como la "percepción de los profesores y de los pares, de los aspectos organizativos y de las condiciones físicas en que se desarrollan las actividades escolares" (p.17). Por otro lado, identifican la posibilidad de que dentro de los climas escolares se pueda encontrar microclimas, y que, a su vez, los climas escolares puedan ser tóxicos o nutritivos.

Los climas escolares percibidos como positivos se han asociado con la disminución de la victimización y la agresión entre pares durante la adolescencia, la mejora del rendimiento académico, del bienestar físico y la promoción de la resiliencia y de comportamientos prosociales (Roffey, 2015).

Para este estudio es de relevancia la promoción del bienestar subjetivo y el desarrollo de la empatía, considerada el principal motivador de comportamientos prosociales en ámbitos educativos. Por tanto, interesa conocer la respuesta empática de un grupo de estudiantes a partir de sus dos componentes: el afectivo y el cognitivo, los cuales inciden en la capacidad del individuo para tomar perspectiva, imaginarse en el lugar de la otredad y generar una respuesta de ayuda de acuerdo con la experiencia emocional que se percibe en otra persona. Los estudios que examinan la respuesta empática en adolescentes son abundantes (Mestre et al., 2004), al igual que las investigaciones sobre clima escolar o bienestar subjetivo (Turner et al., 2014), pero es menos abundante la investigación que integra las tres dimensiones de interés de este estudio.

La motivación principal que condujo esta investigación fue aportar evidencias para que las instituciones educativas valoren otras maneras de consolidar una cultura de paz, de acuerdo con la vivencia subjetiva del clima escolar de la comunidad estudiantil, relacionada con la percepción del apoyo social, el respeto y el bienestar, un enfoque que se considera alternativo a los estudios centrados en la violencia en las escuelas.

En este sentido, a la educación le atañe tanto el bienestar personal como el bienestar colectivo; en consecuencia, el conocimiento de las relaciones entre satisfacción con la vida, la respuesta empática y el clima escolar remite a un interés por el desarrollo socioafectivo en contextos educativos. Se espera que ambientes educativos saludables proporcionen herramientas para la construcción de una sociedad más solidaria, en un momento histórico en el que se ha experimentado la necesidad de conexión afectiva en medio de las dificultades y de la incertidumbre de los tiempos.

\section{REFERENTES CONCEPTUALES}

La revisión de literatura sobre clima escolar permite concluir que se trata de una noción multidimensional. Como señala Miura (2010), este constructo se basa en tres dimensiones: la individual, organizacional y la interpersonal, las cuales interactúan entre sí y le otorgan complejidad a su definición. De este modo, lo objetivo del clima escolar pasa por una interpretación subjetiva, que atribuye un valor a la experiencia de las relaciones en dichos entornos, lo que recuerda la noción de vivencia subjetiva de Vygotski, donde confluyen sentimientos e ideas a partir de la acción humana en relación con otras personas (González, 2013).

La noción de clima escolar se estudia con frecuencia ligada a la comprensión de la violencia escolar, por lo que ha sido objeto de estudio de gran cantidad de investigaciones en relación con la victimización de pares, la intimidación escolar y la agresión, pero también se ha estudiado en relación con el compromiso 
con la institución (Turner et al., 2014), la mejora del rendimiento académico, medidas de bienestar físico y psicológico, y satisfacción con la vida, junto con la mejora de dimensiones de resiliencia (Alfaro et al., 2016; Aron y Milicic, 2013; Berg y Aber, 2015; Berger et al., 2014; Gutiérrez y Gonçalves, 2013; MartínezFerrer et al., 2012; Marshall, 2004; y Pirani et al., 2016). Un poco menos estudiada ha sido su relación con las manifestaciones de comportamientos orientados de manera prosocial; sin embargo, los hallazgos han mostrado que la vivencia de climas escolares positivos trae beneficios en el desarrollo socioemocional y en el desempeño escolar, lo que incide en la manifestación de conductas orientadas a la prosocialidad (Batanova y Loukas, 2014; Roffey, 2015; Ruvalcaba-Romero et al., 2017).

Estos resultados sugieren que los centros educativos, al lado del apoyo familiar, tienen una incidencia en la salud y el bienestar de las personas estudiantes. Por lo que las instituciones educativas están llamadas también a propiciar ambientes donde las personas se sientan seguras, reciban calidez en el ejercicio de la autoridad y apoyo en las dificultades, ya que estos factores se han visto asociados con familias que permiten el desarrollo de comportamientos prosociales en sus hijos e hijas, específicamente en las manifestaciones de comportamiento empático (Alvarado, 2012).

La orientación prosocial del comportamiento se define como toda conducta que la persona realiza voluntariamente y que se dirige a cuidar, ayudar o beneficiar a otra, como la compasión o el altruismo (Holmgren et al.,1998). La empatía es el principal motivador de estas conductas (para mayores referencias puede revisarse Alvarado, 2018), de allí la pertinencia de desarrollar investigaciones que estudien las posibles relaciones entre los climas escolares y los comportamientos prosociales motivados por la empatía.

La empatía fue definida por Eisenberg (1991) como una respuesta fundamentalmente emocional que se despliega de manera coherente cuando se está frente a la manifestación de un estado emocional de otra persona. Este estado emocional que se experimenta guarda una mínima diferenciación entre el sí y la otra persona. De esta definición se destacan dos componentes distintos pero ligados entre sí, por un lado, los procesos cognitivos que permiten tomar distancia o perspectiva ante la situación experimentada por la otra persona; por otro lado, las reacciones emocionales frente al estado emocional.

Los estudios coinciden en que la empatía es un concepto multidimensional, constituido por componentes afectivos y cognitivos. Eisenberg (1991) distingue la toma de perspectiva como comprensión de las cogniciones y estados emocionales de otra persona, a diferencia de la empatía -como preocupación empática-, que se define solo como la reacción emocional frente a la situación de la otra persona. El último aspecto es la simpatía, una respuesta emocional acompañada de sentimientos de tristeza, pero además de preocupación por la otra persona, que le hace orientarse a desear que se sienta mejor; es decir, la persona no solo trata de evitar la experiencia propia del malestar, sino que hay un compromiso con el bienestar de la otra persona.

Para esta autora, las diferencias individuales son un factor que afectan la manera en que se reacciona o la habilidad para regular la propia respuesta ante los estados emocionales que experimenta otra persona, o sea, la posibilidad de experimentar empatía o malestar personal (Eisenberg et al., 1995; Eisenberg et al., 2005).

Con los años se ha desarrollado una amplia investigación al respecto de la empatía en diferentes poblaciones. Con adolescentes se ha explorado en relación con su ambiente educativo y con diferentes características personales en relación con las manifestaciones agresivas y la salud mental.

Los hallazgos han evidenciado que la empatía se asocia con una actitud favorable hacia la autoridad (Moreno et al., 2009) y que la empatía afectiva en personas adolescentes inhibe la agresión (Batanova y Loukas, 2014), lo que parece ser significativo en la prevención de la violencia en las aulas. Esto sugiere que la empatía, comprendida como el factor motivacional de los comportamientos prosociales, actúa sobre el clima escolar y también protege de la depresión (Llorca et al., 2014). Por otra parte, la percepción positiva del clima escolar, junto con altos niveles de preocupación empática, disminuyen la agresión en adolescentes mayores, aunque en preadolescentes solo la preocupación empática aparece asociada con la disminución de la agresión (Batanova y Loukas, 2014). 
De esta manera podría aceptarse que un centro educativo — que vela por mantener las mejores condiciones de aprendizaje, tanto de su enfoque pedagógico como en el cuidado de las relaciones y el bienestar, y que acompaña la formación académica con la formación en justicia social y solidaridad- puede incidir favorablemente en el desarrollo de personas adolescentes con una fuerte preocupación por el bienestar de la otredad, lo que abre la posibilidad de construir mejores condiciones para la consolidación de una cultura de paz en los centros educativos.

El objetivo central que se propuso en esta investigación fue explorar las relaciones entre la percepción del clima escolar en las 5 dimensiones que proponen Aron y Milicic (2013), la respuesta empática, cognitiva y afectiva, como motivador principal de comportamientos que se orientan de manera positiva hacia el bienestar de la otra persona medida por el índice de reactividad interpersonal y la valoración de la satisfacción con la vida de un grupo de adolescentes de educación secundaria. Asimismo, interesaba establecer si existen diferencias por género y edad. La información que aporta este tipo de estudios contribuye en la comprensión de las características de los contextos escolares que pueden contribuir en la prevención de la violencia en los centros educativos (Turner et al., 2014; Villalobos-Parada et al., 2016), además se destaca la contribución de las mismas personas protagonistas al expresar su percepción sobre el clima escolar.

\section{Método}

\section{Diseño, participantes y procedimiento}

Este estudio corresponde a una investigación transversal de tipo correlacional, cuyo objetivo primordial se refiere al interés por explorar si existe alguna asociación entre las variables de estudio; a saber, la respuesta empática, la satisfacción con la vida y la valoración del clima escolar.

Se definió la población participante a partir de un método no probabilístico incidental (Delgado, 2014) de 401 personas, adolescentes de ambos sexos, 170 mujeres y 231 varones. Todas las personas participantes estaban ubicadas en el sistema formal de educación diurna, sus edades oscilaban entre los 15 y 17 años; la media de edad fue de 15.86 ( $D S=0.78$ ). La mayoría de los participantes ( $58.3 \%$ ) vivía con sus madres y padres.

El estudio se presentó como una investigación en el contexto de una formación de doctorado. Se contó con la participación de tres centros educativos públicos de la región metropolitana de Costa Rica y con el consentimiento informado de las familias de las personas participantes que, a su vez, firmaron un asentimiento informado, el cual les fue leído y explicado. El paquete de cuestionarios se cumplimentó en aproximadamente 40 minutos.

\section{Instrumentos de medición}

Los instrumentos seleccionados han sido utilizados y desarrollados fuera del contexto costarricense, por tanto, para garantizar la comprensión de cada uno de los ítems que componen los instrumentos, se realizaron entrevistas cognitivas, según los lineamientos descritos por Smith y Molina (2011), a un grupo de personas adolescentes con las mismas características del conjunto de jóvenes participantes en el estudio. Por otro lado, los instrumentos se sometieron a juicio de expertos, quienes valoraron el grado de comprensión de los reactivos, su pertinencia y relevancia. Para los análisis estadísticos se utilizó el Statistical Pachagefor the Social Sciences (SPSS) versión 22. A cada uno de los instrumentos se le realizó un análisis de homogeneidad de los ítems para valorar su eficacia; los valores utilizados son los propuestos por Delgado (2014), quien define que son aceptablemente homogéneos los ítems que alcanzan un valor mayor a 0.30. 


\section{Satisfacción con la vida}

La medición de la satisfacción con la vida se realizó con la escala de Satisfacción con la vida para estudiantes (SLSS) (Huebner, 1991), que se adaptó de la población chilena por Alfaro et al., (2016). Esta escala cuenta con 7 ítems que se responden de acuerdo con la frecuencia en la que se experimenta la satisfacción en varios aspectos. Las respuestas se pueden dar en 5 niveles tipo Likert, donde 0 es Totalmente en desacuerdo y 4 Totalmente de acuerdo. A partir de los puntajes obtenidos en cada uno de los ítems, se obtiene un índice global de satisfacción con la vida (para mayor detalle puede revisarse el estudio de Alfaro et al., 2016). En este reporte de investigación, la escala alcanzó un coeficiente de confiabilidad de .76, similar a otros reportes de investigación como el de Bluth y Blanton (2013) y Martínez et al. (2007).

\section{Respuesta empática}

Se utilizó el índice de reactividad interpersonal (IRI de Davis, 1980, versión en español de Mestre et al., 2004). Esta escala consta de 28 ítems con respuesta tipo Likert donde el 0 representa el No me describe bien y el 4 Me describe muy bien. La escala fue construida con la intención de valorar dos componentes de la empatía: la empatía afectiva y la cognitiva. A su vez considera cuatro manifestaciones de la empatía; a saber, la toma de perspectiva, la fantasía, la preocupación empática y el malestar personal.

Para esta investigación, el análisis de los reactivos de la escala indicó que 7 ítems no funcionaban adecuadamente, lo que afectó la confiabilidad de la escala. Esos ítems se relacionaban con actividades que la población adolescente en Costa Rica no acostumbra realizar o no está familiarizada, por lo que se procedió a excluirlos. Esto hizo que para el análisis de los datos se considerara una escala de 21 ítems, con un coeficiente de confiabilidad de .84. Se mantuvo la estructura factorial original con los siguientes coeficientes de confiabilidad: fantasía, .76; preocupación empática, .68; toma de perspectiva, .72 y malestar personal, .68.

\section{Percepción del Clima Escolar}

Se utilizó la escala del clima social escolar (ECLIS), desarrollada en población chilena por Aron y Milicic en el año 1999 (Aron et al., 2012). Este instrumento consta de 82 ítems distribuidos en cinco subescalas que valoran diferentes dimensiones de la percepción del clima escolar. Estas se refieren a la percepción que las personas tienen de su relación con el personal docente, compañeras y compañeros de clase, con una breve subescala que indaga sobre la percepción del bullying, y dos subescalas que exploran la satisfacción con el centro educativo y con la infraestructura del este. El cuestionario propone un formato de respuesta tipo Likert con 4 niveles que se refieren al grado de acuerdo con cada una de las afirmaciones de los ítems. Para la subescala Mis profesores, los niveles de respuesta son: todos, la mayoría, pocos o ninguno. Para las subescalas Mis compañeros e Infraestructura, se responde: siempre, casi siempre, pocas veces o nunca; para la subescala de Mi colegio, la persona escoge entre cuatro niveles de acuerdo. La autoría advierte que, por tratarse de un instrumento de varias dimensiones, no se establece un índice global.

Previo a la administración de este instrumento, la evaluación realizada por los jueces expertos, así como en las entrevistas cognitivas, recomendó la utilización de un cuestionario reducido a 75 ítems, ya que algunos de estos eran específicos a la organización de los centros escolares en Chile. El análisis de los ítems, posterior a la administración, indicó que procedía la eliminación de otros reactivos, así que el análisis con pruebas estadísticas se realizó con una versión breve del instrumento constituido por 60 ítems con un alfa de Cronbach de .90. Un análisis de factores fue realizado (análisis de componentes y gráfico de sedimentación), si bien arroja cinco factores que explican el $40.19 \%$ de la varianza total, ofrece una estructura diferente a la 
originalmente propuesta por su autoría, que indica que su propuesta no pudo ser confirmada por un análisis factorial (Aron y Milicic, 2013).

En este estudio, las dimensiones obtenidas fueron Mis Profesores, con una distribución de ítems igual a la versión original, Satisfacción con el colegio, y valoración de la Infraestructura; la cuarta subescala está compuesta por los ítems relacionados a la subescala de Bullying y tres de los ítems de la versión original de la subescala Mis Compañeros. Por último, se obtuvo una nueva dimensión integrada por todos aquellos ítems del cuestionario original, que hacían referencia a las actividades paralelas a la vida académica que promueve la institución, las cuales se han denominado extracurriculares (para mayor detalle de los análisis se recomienda revisar Alvarado, 2018).

\section{Información general}

Se utilizó el cuestionario de información general con preguntas generales acerca de la composición familiar, así como ítems que informan sobre la condición económica familiar, nivel educativo de la persona jefa de hogar, número de integrantes de la familia y zona de procedencia.

\section{Resultados}

Se efectuaron análisis descriptivos y pruebas de hipótesis para confirmar el tipo de pruebas que se realizarían para el análisis de los datos. Debido a que no se cumplió con los criterios de normalidad y homocedasticidad requeridos, fueron elegidas pruebas no paramétricas como la prueba $U$ de Mann-Whitney para muestras independientes y el coeficiente de correlación Rho de Spearman.

\section{Diferencias por edad y sexo}

Los resultados indican que la mayoría de las personas participantes expresan alta satisfacción con la vida (media de 24.51, DS=4.9), sin diferencias significativas por edad o sexo. Respecto del índice de respuesta empática, los resultados indican que las personas participantes obtienen una puntuación moderada de 2.99 (media por elemento). Si bien no se presentan diferencias significativas por edad, sí se obtienen diferencias significativas por género, lo que indica que las mujeres presentan mayor tendencia a dar una respuesta empática en las cuatro dimensiones del IRI, lo que coincide con otras investigaciones (sobre este punto puede revisarse a Mestre et al., 2004). Para las dimensiones del clima escolar no se pueden confirmar diferencias por edad, pero sí diferencias significativas por género en tres de las subescalas. En la subescala de Bullying se interpreta que la percepción de los participantes varones tiende a identificar mayor presencia de estas conductas de matonismo de parte de sus pares, como burlarse, pelear, o hacer sufrir. En las subescalas de percepción de las Condiciones de infraestructura y en Actividades extracurriculares, las mujeres reconocen mayor acuerdo con las afirmaciones contenidas en estas subescalas. Estos resultados coinciden con las correlaciones obtenidas entre dichas variables (Tabla 1). 
TABLA 1.

Correlaciones no paramétricas entre variable género, SSLSS, IRI y Clima escolar

\begin{tabular}{|c|c|c|c|c|c|c|c|c|c|c|c|c|}
\hline & & Sexo & SLSS IRI & $\mathrm{P}$ & IE & SC. & $\mathrm{AE}$ & B & $\mathrm{F}$ & $\mathrm{PE}$ & $\mathrm{TP}$ & MP \\
\hline Rho de Spearman Sex & Coeficiente correlación & 000 & $094-.294$ & & $6-.141^{k * k}$ & -.003 & $-.135^{*}$ & $194^{* * x}$ & $-.184^{* * *}$ & $-.236^{* * *}$ & $-.103^{x}$ & $-.235^{* *}$ \\
\hline & $\begin{array}{l}\text { Sig. (bilateral) } \\
\mathrm{N}\end{array}$ & 401 & $\begin{array}{l}.065 .000 \\
386343\end{array}$ & & $\begin{array}{l}2.007 \\
9362\end{array}$ & $\begin{array}{l}.956 \\
355\end{array}$ & $\begin{array}{l}.011 \\
350\end{array}$ & $\begin{array}{l}.000 \\
369\end{array}$ & $\begin{array}{l}.000 \\
386\end{array}$ & $\begin{array}{l}.000 \\
376\end{array}$ & $\begin{array}{l}.042 \\
390\end{array}$ & $\begin{array}{l}.000 \\
383\end{array}$ \\
\hline
\end{tabular}

Fuente: elaboración propia.

Nota: SLSS: cuestionario de satisfacción con la vida. IRI: índice de reactividad interpersonal. Subescalas de clima escolar: P: profesores. IE: infraestructura. SC: satisfacción con el colegio. AE: actividades extracurriculares. B: bullying. F: fantasía. PE: preocupación empática. TP: toma de perspectiva. MP: malestar personal.

\section{Análisis de correlación no paramétrica}

Se realizó el análisis de correlación Rho de Spearman para las dimensiones Satisfacción con la vida, Respuesta empática (IRI) y las cinco dimensiones evaluadas en el cuestionario de clima escolar (Tabla 2).

Para el Índice de satisfacción con la vida se obtuvo una asociación significativa con todas las dimensiones del cuestionario del clima escolar, excepto con la subescala de Bullying. La asociación más alta se obtuvo con la subescala Mis profesores (.339, $p<.01)$, seguida de la Satisfacción con el colegio $(.247, p<.01)$, la percepción de las Condiciones de infraestructura $(.237, p<.01) \mathrm{y}$, finalmente, con la percepción de las Actividades extracurriculares $(.140, p<.01)$ que ofrece el centro educativo. Asimismo, se observa una asociación positiva con Toma de perspectiva $(.152, p<.01)$, evaluada en el IRI, uno de los componentes más importantes de la empatía que, de acuerdo con Mestre et al. (2004), junto con la Preocupación empática, "son responsables de una respuesta empática más madura basada en la comprensión del otro y en compartir su estado emocional" (p.258).

Con relación al Índice de respuesta empática (índice de reactividad interpersonal), la asociación es significativa con las dimensiones de clima escolar: Profesores $(.131, p<.05)$, Infraestructura $(.199, p<.01)$, Satisfacción con el colegio $(.245, p<.01)$, Actividades extracurriculares $(.121, p<.05)$; excepto con la dimensión de Bullying. A su vez, presenta una asociación significativa entre todas las subescalas que lo componen.

Respecto a la percepción del clima escolar, los resultados que no han sido mencionados se presentan por subescala. La subescala Mis profesores, presenta una asociación positiva y significativa con la subescala de Toma de perspectiva $(.302, p<.01)$. La subescala Condiciones de infraestructura, se asocia además con tres subescalas del IRI: Fantasía $(.119, p<.01)$, Preocupación empática $(.133, p<.01)$ y Toma de perspectiva $(.251, p<.01)$. Cabe hacer notar que la subescala Satisfacción con el colegio presenta una asociación significativa no solo con los dos índices de este estudio, sino que además se correlaciona significativamente con cada una de las cuatro subescalas del IRI, Toma de perspectiva $(.320, p<.01)$, Preocupación empática $(.145, p<.01)$, Fantasía $(.123$, $p<.05)$, y es la única dimensión de la percepción del clima escolar que guarda una asociación significativa con Malestar personal $(.175, p<.01)$. La subescala Actividades extracurriculares se correlaciona con la subescala Toma deperspectiva $(.152, p<.01)$. La subescala sobre comportamientos de matonismo o bullying, no presenta correlaciones significativas con las dimensiones de trabajo en este estudio.

Finalmente, se observan correlaciones significativas entre las dimensiones del cuestionario Clima escolar, excepto con la dimensión que evalúa la percepción del bullying, lo que puede ser un indicador de que este instrumento mide la satisfacción global de las personas con su institución y podría trabajarse sin la dimensión de matonismo, más que como fin diagnóstico de las conductas agresivas o violentas en el centro de enseñanza. 
TABLA 2.

Correlaciones no paramétricas entre las variables principales del estudio

\begin{tabular}{|c|c|c|c|c|c|c|c|c|c|c|c|c|c|}
\hline \multirow{5}{*}{\multicolumn{2}{|c|}{$\begin{array}{c}\text { Rho de Spearman SLSS } \\
\text { IRI }\end{array}$}} & & SLSS & IRI & $\mathrm{P}$ & IE & SC. & $\mathrm{AE}$ & B & F & PE & TP & MP \\
\hline \multirow{21}{*}{ Rho de Spearman } & & Coeficiente correlación & 1.000 & .077 & $339^{* *}$ & $.237^{* * *}$ & $247^{* * *}$ & $.140^{* / *}$ & .093 & -.013 & .056 & $.152^{* * *}$ & .025 \\
\hline & & $\begin{array}{l}\text { Sig. (bilateral) } \\
\text { N }\end{array}$ & 386 & $\begin{array}{l}.162 \\
330\end{array}$ & $\begin{array}{l}.000 \\
307\end{array}$ & $\begin{array}{l}.000 \\
349\end{array}$ & $\begin{array}{l}.000 \\
342\end{array}$ & $\begin{array}{l}.010 \\
338\end{array}$ & $\begin{array}{l}.082 \\
354\end{array}$ & $\begin{array}{l}.798 \\
371\end{array}$ & $\begin{array}{l}.283 \\
363\end{array}$ & $\begin{array}{l}.003 \\
375\end{array}$ & $\begin{array}{l}.635 \\
368\end{array}$ \\
\hline & & Coeficiente correlación & & 1.000 & $.131^{*}$ & $.199^{* * *}$ & $.245^{* * *}$ & $.121^{*}$ & -.003 & $.751^{* *}$ & $.763^{* *}$ & \multirow{2}{*}{\multicolumn{2}{|c|}{$\begin{array}{ll}.687^{* * *} & .657^{* * *} \\
.000 & .000 \\
343 & 343\end{array}$}} \\
\hline & & $\begin{array}{l}\text { Sig. (bilateral) } \\
\text { N }\end{array}$ & & 343 & $\begin{array}{l}.028 \\
280\end{array}$ & $\begin{array}{l}.000 \\
314\end{array}$ & $\begin{array}{l}.000 \\
309\end{array}$ & $\begin{array}{l}.035 \\
305\end{array}$ & $\begin{array}{l}.963 \\
321\end{array}$ & $\begin{array}{l}.000 \\
343\end{array}$ & $\begin{array}{l}.000 \\
343\end{array}$ & & \\
\hline & $\mathrm{P}$ & Coeficiente correlación & & & 1.000 & $.473^{k-k}$ & $499^{* * *}$ & $.456^{* *}$ & .046 & .018 & .046 & \multicolumn{2}{|c|}{$.302^{* *} .101$} \\
\hline & & $\begin{array}{l}\text { Sig. (bilateral) } \\
\mathrm{N}\end{array}$ & & & 319 & $\begin{array}{l}.000 \\
293\end{array}$ & $\begin{array}{l}.000 \\
286\end{array}$ & $\begin{array}{l}.000 \\
284\end{array}$ & $\begin{array}{l}.425 \\
300\end{array}$ & $\begin{array}{l}.746 \\
310\end{array}$ & $\begin{array}{l}424 \\
300\end{array}$ & $\begin{array}{l}.000 \\
314\end{array}$ & $\begin{array}{l}.077 \\
309\end{array}$ \\
\hline & IE & Coeficiente correlación & & & & 1.000 & $478^{* * *}$ & $.385^{* * *}$ & -.031 & $.119^{*}$ & $.133^{*}$ & $.251^{* * *}$ & .091 \\
\hline & & $\begin{array}{l}\text { Sig. (bilateral) } \\
\text { N }\end{array}$ & & & & 362 & $\begin{array}{l}.000 \\
331\end{array}$ & $\begin{array}{l}.000 \\
325\end{array}$ & $\begin{array}{l}.572 \\
341\end{array}$ & $\begin{array}{l}.026 \\
349\end{array}$ & $\begin{array}{l}.014 \\
343\end{array}$ & $\begin{array}{l}.000 \\
352\end{array}$ & $\begin{array}{l}.090 \\
347\end{array}$ \\
\hline & SC & Coeficiente correlación & & & & & 1.000 & $.392^{* *}$ & .036 & $.123^{*}$ & $.145^{* *}$ & $.320^{* *}$ & $.175^{* * *}$ \\
\hline & & $\begin{array}{l}\text { Sig. (bilateral) } \\
\mathrm{N}\end{array}$ & & & & & 355 & $\begin{array}{l}.000 \\
316\end{array}$ & $\begin{array}{l}.513 \\
331\end{array}$ & $\begin{array}{l}.023 \\
343\end{array}$ & $\begin{array}{l}.008 \\
337\end{array}$ & $\begin{array}{l}.000 \\
346\end{array}$ & $\begin{array}{l}.001 \\
341\end{array}$ \\
\hline & $\mathrm{AE}$ & Coeficiente correlación & & & & & & 1.000 & .068 & .082 & .078 & \multicolumn{2}{|c|}{$.157^{* * *} .069$} \\
\hline & B & $\begin{array}{l}\text { Sig. (bilateral) } \\
\mathrm{N} \\
\text { Coeficiente correlación }\end{array}$ & & & & & & 350 & $\begin{array}{l}.220 \\
332\end{array}$ & $\begin{array}{l}.134 \\
336 \\
-053\end{array}$ & $\begin{array}{l}.155 \\
334 \\
005\end{array}$ & \multicolumn{2}{|c|}{$\begin{array}{ll}.004 & .207 \\
340 & 337\end{array}$} \\
\hline & & $\begin{array}{l}\text { Sig. (bilateral) } \\
\mathrm{N}\end{array}$ & & & & & & & 369 & $\begin{array}{l}.316 \\
356\end{array}$ & $\begin{array}{l}.922 \\
349\end{array}$ & $\begin{array}{l}.834 \\
361\end{array}$ & $\begin{array}{l}.121 \\
355\end{array}$ \\
\hline & $\mathrm{F}$ & Coeficiente correlación & & & & & & & & 1.000 & $379^{* *}$ & $.337^{* *}$ & $.372^{* * *}$ \\
\hline & & $\begin{array}{l}\text { Sig. (bilateral) } \\
\text { N }\end{array}$ & & & & & & & & 386 & $\begin{array}{l}.000 \\
362\end{array}$ & $\begin{array}{l}.000 \\
378\end{array}$ & $\begin{array}{l}.000 \\
370\end{array}$ \\
\hline & P E & Coeficiente correlación & & & & & & & & & 1.000 & $.462^{* * *}$ & $.378^{*-k}$ \\
\hline & & $\begin{array}{l}\text { Sig. (bilateral) } \\
\text { N }\end{array}$ & & & & & & & & & 376 & $\begin{array}{l}.000 \\
366\end{array}$ & $\begin{array}{l}.000 \\
360\end{array}$ \\
\hline & $\mathrm{TP}$ & Coeficiente correlación & & & & & & & & & & 1.000 & $.231^{* * *}$ \\
\hline & & $\begin{array}{l}\text { Sig. (bilateral) } \\
\mathrm{N}\end{array}$ & & & & & & & & & & \multirow[t]{2}{*}{390} & $\begin{array}{l}.000 \\
377\end{array}$ \\
\hline & MP & Coeficiente correlación & & & & & & & & & & & 1.000 \\
\hline & & $\begin{array}{l}\text { Sig. (bilateral) } \\
\text { N }\end{array}$ & & & & & & & & & & \multicolumn{2}{|c|}{383} \\
\hline
\end{tabular}

Fuente: elaboración propia

${ }^{* * *}$ La correlación es significativa en el nivel 0.01 ( 2 colas)

Nota: SLSS: cuestionario de satisfacción con la vida. IRI: índice de reactividad interpersonal. Subescalas de clima escolar: P: profesores. IE: infraestructura. SC: satisfacción con el colegio. AE: actividades extracurriculares. B: bullying. Subescalas del IRI: F: fantasía. PE: preocupación empática. TP: toma de perspectiva. MP: malestar personal.

\section{Discusión}

Los estudios de tipo longitudinal muestran que la vivencia de satisfacción con la vida, cuando se es joven, representa un antecedente significativo para el subsecuente desarrollo personal e interpersonal. Las investigaciones reportan relaciones estadísticamente significativas entre altos niveles de satisfacción con la vida y mayor compromiso escolar, buenas relaciones familiares y con pares, así como una disminución de los problemas de conducta. En este sentido, un hallazgo importante del estudio son los valores de satisfacción 
con la vida moderadamente altos obtenidos en la mayoría de la población entrevistada, resultados que coinciden con otros estudios de habla hispana (Urrelo y Huamani, 2019) y con resultados de investigación con población estadounidense, que señalan que, tanto menores de edad como adultos, reportan niveles moderadamente altos de satisfacción con la vida y solo un $10 \%$ de personas jóvenes refieren insatisfacción (Lyons et al., 2016). En cuanto a las diferencias en la satisfacción con la vida por género, los resultados no son concluyentes (Arias et al., 2018).

Por otra parte, en este estudio la satisfacción con la vida se asoció con todas las dimensiones del clima escolar (la asociación más alta con la subescala Mis profesores) y una asociación positiva con Toma de perspectiva del IRI. Este hallazgo es similar a los obtenidos en otras investigaciones que han evidenciado que experimentar satisfacción con la vida, también se relaciona con una experiencia positiva en la institución educativa (Gutiérrez y Gonçalves, 2013; Linden, 2012; Turner et al., 2014; Martínez et al., 2007; Ruus et al. 2007; Villalobos et al., 2016). Como señalan Gutiérrez y Gonçalves (2013), aún no está clara la dirección de la relación entre experimentar satisfacción con la vida y satisfacción con la institución educativa; sin embargo, en su estudio la satisfacción con el centro educativo predijo la satisfacción con la vida en adolescentes.

Este es un resultado importante porque al mismo tiempo muestra la responsabilidad compartida entre la familia y la institución educativa en el bienestar de las poblaciones jóvenes. Además, durante la adolescencia el centro educativo puede llegar a satisfacer necesidades de interacción de gran valor para el proceso de desarrollo, donde se consolidan aspectos relacionados con la identidad y la apertura del mundo familiar. De allí también la importancia de promover que las personas se mantengan dentro del sistema educativo, más allá del aprendizaje de contenidos, ya que, según Lyons et al. (2016), los factores ambientales son predictores significativos de satisfacción con la vida, aún más que los rasgos de personalidad.

$\mathrm{Al}$ respecto de la satisfacción con el centro educativo, esta es considerada un dominio de la satisfacción global con la vida en personas jóvenes, además se refiere a una evaluación que implica tanto procesos cognitivos como afectivos, por lo que se considera un valioso indicador de bienestar subjetivo en el contexto escolar (Huebner, 2004), lo que podría explicar su asociación con las dimensiones del clima escolar que se examinaron en este estudio y con la dimensión toma de perspectiva del índice de respuesta empática como componente cognitivo de la empatía, que, como se ha indicado, permite tomar distancia o perspectiva ante la situación experimentada por la otra persona, un paso necesario para el desarrollo del comportamiento prosocial empático.

La satisfacción con el profesorado, como dimensión del clima escolar, es un aspecto que ha sido destacado en otras investigaciones. El respeto mutuo entre docentes y estudiantado, así como el que este se sienta valorado y aceptado en condiciones de confianza y apoyo, se asocian con percepciones de clima escolar positivo, ya que, como indican Martínez-Ferrer et al. (2012), se "configura un espacio de interacción social positivo que favorece la vinculación del alumno con la escuela y el profesorado y previene la agresión escolar" (p.875). La satisfacción con la vida, en este estudio, muestra también asociaciones significativas con las actividades extracurriculares y las condiciones de infraestructura, estos resultados constituyen un aporte para que los centros educativos cuenten con criterios para evaluar las condiciones que ofrecen a la comunidad educativa para llevar a cabo los aprendizajes. No solo la condición de infraestructura de las instituciones es un tema de gran valor, sino también las oportunidades de desarrollo personal que se pueden propiciar a partir de la oferta de actividades extracurriculares, que se relacionan con el bienestar emocional y físico de las poblaciones.

Por otro lado, la asociación positiva entre la dimensión de percepción de las condiciones de la infraestructura de la institución con el índice de empatía y las dimensiones que lo componen -fantasía, preocupación empática y toma de perspectiva, que a su vez se asocia a la satisfacción con la instituciónsugiere que el descuido o la negligencia en el cuidado de la planta física de las instituciones puede afectar tanto la satisfacción con la institución como las condiciones de convivencia escolar y su impacto en la promoción de comportamientos orientados positivamente. Otros estudios han reportado que las condiciones del ambiente 
escolar tienen un impacto sobre la motivación y el aprendizaje de las personas estudiantes (Asiyai, 2014). $\mathrm{Al}$ respecto, Aron y Milicic (2013) llamaron la atención acerca de la relevancia para la convivencia de "los espacios armoniosos, gratos, bien iluminados y con temperatura adecuadas (que) generan una sensación de bienestar. Mientras que los ambientes fríos, descuidados y deteriorados, inducen en los alumnos y profesores estados de ánimo que obstaculizan el rendimiento y la convivencia" (p.60).

Respecto al índice de reactividad interpersonal, en relación con la variable género, se confirma que las mujeres tienden a presentar una mayor respuesta empática que los varones, tanto en el índice global del IRI como en cada una de sus dimensiones. Estas diferencias se han atribuido, en parte, a las distintas experiencias en la socialización de hombres y mujeres, tanto en el contexto familiar como en el escolar en relación con las conductas de ayuda y de cuidado ante la necesidad de las demás personas, ya que a las mujeres, desde pequeñas, se les enseña a cuidar de los demás (Batanova y Loukas, 2014).

El índice de reactividad interpersonal también obtuvo asociaciones significativas con las dimensiones del clima escolar (mis profesores, condiciones de infraestructura, satisfacción con la institución y actividades extracurriculares), excepto con la dimensión que evalúa el bullying, un resultado que no sorprende, ya que las conductas de acoso y matonismo son antagónicas de un comportamiento empático, que se espera que inhiba el comportamiento agresivo (Batanova y Loukas, 2014; Castro et al., 2019; Riekie et al., 2017). Sin embargo la relación entre el Índice de Reactividad Interpersonal (IRI) y el Clima Escolar en términos de causalidad aún no se ha definido con claridad (Batanova y Loukas, 2016).

No obstante, los resultados obtenidos animan a pensar que las instituciones educativas, que generan alta satisfacción entre su estudiantado, podría propiciar mejores condiciones para expresar un comportamiento empático, de cuidado y de respeto por la situación de otras personas, modelado, a su vez, en las interacciones cotidianas con el profesorado y el cuidado del centro educativo, por su infraestructura y por los programas diseñados para impactar el bienestar de sus poblaciones. Esto vendría a ser un comportamiento prosocial en retribución al trato que la institución educativa le ofrece.

Es importante resaltar el hallazgo acerca de las diferencias por género encontradas en la percepción de conductas de hostigamiento o bullying en la institución educativa. Posiblemente esto se debe a que la presencia de agresión abierta es mayor entre los hombres que en las mujeres, lo que coincide con otros reportes de investigación (Loukas y Robinson, 2004). En esta línea, Cerezo (2008) mostró que los varones presentan mayor incidencia de conductas agresivas, además reportaron una situación agresiva casi diaria, mientras que las mujeres consideran que una situación agresiva ocurría una o dos veces por semana, lo que también parece estar relacionado con los patrones de socialización de género. Por otro lado, la percepción de las conductas de matonismo en una institución educativa es una fuente de información respecto de la satisfacción del estudiantado con su centro educativo, lo que proporciona información importante para quienes dirigen la institución.

\section{Conclusiones}

1. Los resultados de esta investigación confirman una asociación significativa entre las variables satisfacción con la vida, clima escolar y el índice de reactividad interpersonal, lo que satisface el objetivo central de investigación.

2. Es necesario que las instituciones educativas reflexionen sobre su papel en la socialización de las prácticas culturales y de género que diferencian lo masculino y lo femenino en la manifestación de comportamientos de cuido, de ayuda y de preocupación por el otro, prácticas que han perpetuado una distribución de tareas asociadas con el género. En este sentido, la promoción de relaciones libres de violencia en ambientes educativos, así como en el planteamiento de propuestas que se orienten hacia formas de convivencia más armoniosas, deberán visibilizar estas diferencias, ya que para alcanzar el éxito en estos programas parece importante abordar el tema del comportamiento 
empático, no solo desde la toma de perspectiva como dimensión cognitiva, sino también desde la preocupación empática, como dimensión afectiva, y desarrollar la capacidad de imaginarse en el lugar de la otredad, desde lo que significa para cada persona cuidar y comprometerse con quien sufre, sentir el dolor de la otra persona y ayudarle.

3. El cuestionario clima escolar (ECLIS), revisado para Costa Rica, proporciona información relevante al respecto de la percepción de la población joven sobre su clima escolar. En esta investigación se establecieron cinco dimensiones de análisis: la dimensión Mis profesores (original de Aron y Milicic, 2012), la dimensión Mi colegio como satisfacción global con la institución; las dimensiones sobre percepción del bullying, condiciones de infraestructura, y la dimensión actividades extracurriculares. La información que este tipo de estudios ofrece sobre la satisfacción de la comunidad estudiantil con sus centros educativos, se considera de gran valor para la consecución de una convivencia caracterizada por el respeto mutuo, la confianza, la seguridad y el apoyo, que, como se ha descrito, son valores que contribuyen con el bienestar de las personas, para que se sientan bien consigo mismas y logren comprometerse con el bienestar de las demás.

4. Asimismo, el estudio permite poner el acento en la importancia de la relación de las personas estudiantes con sus docentes, así como el valor que pueden tener las condiciones de infraestructura y las actividades extracurriculares en el bienestar de la comunidad estudiantil, especialmente cuando se atienden poblaciones en riesgo social, para quienes las instituciones educativas podrían representar el único lugar donde estas personas puedan sentirse cuidadas y valoradas en un momento de la vida en que las interacciones sociales son de suma importancia para la construcción de su identidad personal.

5. El estudio confirma que la dimensión de bullying no presenta asociación con el índice de satisfacción con la vida como medida del bienestar subjetivo ni con las formas de respuesta prosocial. En este sentido, las instituciones educativas deberán procurar un ambiente libre de violencia, para lo cual, el papel de la persona docente como líder educativo es de suma importancia para salvaguardar los derechos de las personas menores de edad en sus espacios de aprendizaje, que inspiren no solo un compromiso con el proyecto educativo y la institución, sino también con la comunidad estudiantil; esto implica no tolerar conductas agresivas que se legitiman por la socialización de género y que en ocasiones pasan desapercibidas en los salones de clase.

6. De manera general, se considera que las instituciones educativas tienen dos funciones muy claras e importantes para la sociedad, una relacionada con la enseñanza de nuevos conocimientos que forman parte de la cultura universal y otra compartida con la familia en la socialización secundaria de las poblaciones más jóvenes. Ninguna otra instancia logra de manera sistemática y a partir de valores explícitos este importante papel social. No obstante, aunque no tan explícito, la institución educativa tiene otra función relacionada con el desarrollo y el bienestar subjetivo de su alumnado, lo que la hace compartir una responsabilidad social en el área de la salud mental. En este sentido, la vida social en las instituciones educativas es un ejercicio para prepararse para la vida en sociedad, pero al mismo tiempo sienta bases para el sano desarrollo de sus poblaciones, lo que se refleja en las pautas de convivencia.

7. La confiabilidad y las evidencias de validez, obtenidas al respecto de los instrumentos utilizados en esta investigación, indican que se trata de instrumentos confiables para continuar su uso en procesos de investigación en este campo en diferentes contextos educativos del país. En particular, el cuestionario de clima escolar deberá someterse a confirmación para obtener mayores evidencias de su estructura factorial en la población costarricense. Sin embargo, se considera que la versión utilizada en este estudio proporciona fuertes evidencias de su validez para continuar utilizándolo. Por tanto, este estudio constituye una base para el desarrollo de investigación dirigida 
a profundizar el conocimiento de los climas escolares y su relación con la empatía y el bienestar de la comunidad educativa.

Sobre las limitaciones de este estudio, si bien se obtuvo información relevante al respecto de la relación entre satisfacción con la vida, clima escolar y respuesta empática, el tipo de investigación correlacional impide establecer relaciones de causalidad entre las variables aquí estudiadas, lo que limita los alcances de estas conclusiones. Asimismo, el proceso de selección de la población participante no permite realizar generalizaciones al respecto de los resultados obtenidos. Futuras investigaciones podrían profundizar el estudio de las dimensiones de esta investigación al ampliar la consulta al personal docente y su relación con la satisfacción laboral y el tamaño de la muestra, así como la representación de otro tipo de centros educativos y de diferentes regiones del país.

\section{RECOMENDACIONES}

Se reconocen tres diferentes ámbitos de acción para fortalecer climas escolares positivos y comportamientos prosociales. Al sistema educativo, como un macro nivel, le corresponde emitir políticas que acompañen las acciones de las instituciones educativas en materia de construcción de una cultura de paz, los centros educativos y las familias.

En la institución educativa, el personal directivo está llamado a reconocer su papel como líder en el desarrollo socioemocional de la comunidad estudiantil y en el acompañamiento de su personal, al propiciar condiciones de trabajo y educativas que fortalezcan los lazos de comunidad en un ambiente seguro. Para alcanzar este objetivo, es importante que la institución abra espacios para conocer la satisfacción de la comunidad educativa con el centro de enseñanza, para esto se sugiere la utilización del cuestionario ECLIS (versión revisada para Costa Rica).

Además, se recomienda desarrollar intervenciones psicopedagógicas dirigidas a la población estudiantil, profesorado y familias en el tema del comportamiento prosocial y socialización de género. Así como la formulación de proyectos que promuevan actividades extracurriculares dirigidas a la satisfacción con la vida

y la felicidad de las poblaciones estudiantiles al permitir encuentros sociales, desarrollo de habilidades para la vida y un lugar seguro donde pasar un tiempo fuera de casa.

\section{REFERENCIAS BIBLIOGRÁFICAS}

Alfaro, J., Guzmán, J., Reyes, F., García, C., Varela, J.y Sirlopú, D. (2016). Satisfacción Global con la Vida y Satisfacción Escolar en Estudiantes Chilenos. PSYKHE, 25(2), 1-14. https://bit.ly/3DzufWJ

Alvarado, K. (2012). Empatía y clima familiar en niños costarricenses de edad escolar. Revista Electrónica Actualidades Investigativas en Educación, 12(3), 1-27. https://www.redalyc.org/articulo.oa?id=44723985002

Alvarado, K. (2018). Clima escolar y bienestar subjetivo en adolescentes de educación secundaria: El rol de la empatía en el salón de clase [tesis doctoral]. Universidad Estatal a Distancia de Costa Rica. https://repositorio.uned.ac.cr/ handle/120809/1804

Arias, W., Huamani, C. y Caycho, T. (2018). Satisfacción con la vida en escolares de la ciudad de Arequipa. Propósitos y Representaciones, 6(1), 1-22. https://doi.org/10.20511/pyr2018.v6n1.206

Aron, A. y Milicic, N. (2013). Clima social escolar. Escalas para su evaluación Trillas.

Aron, A., Milicic, N. y Armijo, I. (2012). Clima Social Escolar: una escala de evaluación -Escala de Clima Social Escolar, ECLIS. Universitas Psychologica, 11(3), 803-813. https://www.redalyc.org/pdf/647/64724634010.pdf

Asiyai, R. (2014). Students' perception of the condition of their classroom physical learning environment and its impact on their learning and motivation [Percepción de estudiantes de la condición de su ambiente físico de clase y su impacto sobre su aprendizaje y la motivación]. College Student Journal, 48(4), 
716-726. http://search.ebscohost.com.ezproxy.sibdi.ucr.ac.cr:2048/login.aspx?direct=true $\& d b=a s n \& A N=10$ 0358667\&lang $=$ es\&site $=$ ehost-live\&scope $=$ site

Batanova, M. y Loukas, A. (2014). Unique and Interactive Effects of Empathy, Family, and School Factors on Early Adolescents' Aggression [Efectos únicos e interactive de los factores de empatía, familia y escuela sobre la aggression en la adolescencia temprana].J. Youth Adolescence, 43, 1890-1902. https://doi.org/10.1007/s1096 4-013-0051-1

Berg, J.y Aber, J. (2015). A multilevel view of predictors of children's perceptions of school interpersonal climate [Una vision multinivel de los predictores de las percepciones de los niños sobre el clima escolar interpersonal].Journal of Educational Psychology, 107(4), 1150-1170. https://doi.org/10.1037/edu0000027

Berger, C., Álamos, P., Milicic, N. y Alcalay, L. (2014). Rendimiento académico y las dimensiones personal y contextual del aprendizaje socioemocional: evidencias de su asociación en estudiantes chilenos. Universitas Psychologica, 13(2), 627-635. http://www.scielo.org.co/pdf/rups/v13n2/v13n2a19.pdf

Bluth, K. y Blanton, P. (2013). Mindfulness and Self-Compassion: Exploring Pathways to Adolescent Emotional WellBeing [Mindfulness y autocompasión: Explorando caminos hacia el bienestar emocional de los adolescentes]. Journal of Child y Family Studies, 23, 1298-1309. https://doi.org/10.1007/s10826-013-9830-2

Castro, M., Zurita-Ortega, F., Ruiz-Rico, GR-R., Chacón-Cuberos, R. (2019). Explanatory model of violent behaviours, self-concept and empathy in schoolchildren. Structural equations analysis. PLoS ONE, 14(8), 1-15. https://doi.org/10.1371/journal.pone.0217899

Cerezo, F. (2008). Agresores y víctimas del bullying. Desigualdades de género en la violencia entre escolares. Información Psicológica, 94, 49-59. https://dialnet.unirioja.es/servlet/articulo?codigo $=2857916$

Delgado, C. (2014). Viajando a Ítaca por mares cuantitativos. Manual de ruta para investigar en grado y postgrado. Amarú.

Eisenberg, N. (1991). Values, Sympathy, and Individual Differences: toward a Pluralism of Factors Influencing Altruism and Empathy [Valores, simpatía y diferencias individuales: hacia un pluralismo de factores que influencian el altruismo y la empatía]. Psychological Inquiry, 2(2), 128-131. https://doi.org/10.1207/s153279 65 pli0202_5

Eisenberg, N., Carlo, G., Murphy, B. y Van Court, P. (1995). Prosocial Development in Late Adolescence: A Longitudinal Study. Child Development, 66, 1179-1197. https://doi.org/10.2307/1131806

Eisenberg, N., Cumberland, A., Guthrie, I., Murphy, B. y Shepard, S. (2005). Age Changes in Prosocial Responding and Moral Reasoning in Adolescence and Early Adulthood. Journal of Research on Adolescence, 15(3), 235-260. https://doi.org/10.1111/j.1532-7795.2005.00095.x

González, F. (2013). La subjetividad en una perspectiva cultural histórica: avanzando sobre un legado inconcluso. Revista de CS, (11), 19-42. http://www.scielo.org.co/pdf/recs/n11/n11a02.pdf

Gutiérrez, M. y Gonçalves, T-O. (2013). Activos para el desarrollo, ajuste escolar y bienestar subjetivo de los adolescentes. International journal of psychology and psychological therapy, 13(3), 339-355. https://dialnet.uniri oja.es/servlet/articulo?codigo $=4420912$

Holmgren, R., Eisenberg, N. y Fabes, R. (1998). The Relations of Children's Situational Empathy-related Emotions to Dispositional Prosocial Behaviour [Las relaciones de las emociones relacionadas con la empatía situacional de niños con la conducta prosocial disposicional]. International Journal of Behavioral Development, 22(1), 169193. https://doi.org/10.1080/016502598384568

Huebner, E. (1991). Further Validation of the Students Life Satisfaction Scale: The Independence of Satisfaction and Affect Ratings [Más allá de la validación de la escala de satisfaccion con la vida de estudiantes. Independencia de las calificaciones de satisfacción y afecto]. Journal of Psychoeducational Assesment, 9, 363-368. https://doi.org/ $10.1177 / 073428299100900408$

Huebner, E. (2004). Research on Assessment of Life Satisfaction of Children and Adolescents, Social Indicators Research, 66(1-2).3-33. http://www.jstor.org.ezproxy.sibdi.ucr.ac.cr:2048/stable/27522057 
Linden, A. (2012). Situational and intrapersonal predictors of school and life satisfaction of elementary school students [Predictores situacionales e intrapersonales de la satisfacción escolar y con la vida de estudiantes de primaria] [Tesis doctoral]. Universidad de Fordham, New York. USA.

Llorca, A., Mesurado, B. y Samper, P. (2014). El rol mediador de la empatía, la conducta prosocial y la conducta agresiva en la depresión y la ansiedad. Ansiedad y Estrés, 20(2-3), 247-258. https://bit.ly/3C7Xy1M

López, V., Bilbao, M., Ascorra, P., Moya, I. y Morales, M. (2014). Escala de Clima Escolar: adaptación al español y validación en estudiantes chilenos. Revista Universitas Psychologica, 13(2), 15-25. http://www.redalyc.org/pdf 1647/64733438025.pdf

Loukas, A. y Robinson, S. (2004). Examining the Moderating Role of Perceived School Climate in Early Adolescent Adjustment. Journal of Research on Adolescence, 14(2), 209-233. https://doi.org/10.1111/j.1532-7795.2004.0 1402004.x

Lyons, M., Huebner, E. y Hills, K. (Jun, 2016). Relations among Personality Characteristics, Environmental Events, Coping Behavior and Adolescents' Life Satisfaction. Journal of Happiness Studies, 17(3), 1033-1050. https://d oi.org/10.1007/s10902-015-9630-z

Marshall, M. (2004). Examining School Climate: Defining Factors and Educational Influences [Explorando el clima escolar: Definiendo factores e influencias edicativas]. (versión electronica). Center for Research on School Safety, School Climate and Classroom Management. Georgia State University Center for School Safety, School Climate and Classroom Management. https://www.researchgate.net/publication/237376259_Examining_School_Cli mate_Defining_Factors_and_Educational_Influences

Martínez, M., Buelga, S. y Cava, M. (2007). La satisfacción con la vida en la adolescencia y su relación con la autoestima y el ajuste escolar. Anuario de Psicología, 38(2), 293-303. http://www.raco.cat/index.php/AnuarioPsicologia/a rticle/view/74205/94371

Martínez-Ferrer, B., Povedano-Díaz, A., Amador-Muñoz, L. y Moreno-Ruiz, D. (2012). Clima escolar, satisfacción con la vida y victimización en la escuela. Un análisis del efecto moderador del género. Anales de psicología, 28(3), 875-882. https://doi.org/10.6018/analesps.28.3.156121

Mestre, V., Frías, M. y Samper, P. (2004). La medida de la empatía: análisis del Interpersonal Reactivity Index. Psicothema, 16(2), 255-260. http://www.psicothema.com/psicothema.asp?id=1191

Miura, T. (2010). School Climate: Development of a Comprehensive Definition [Clima escolar: Desarrollo de una definición comprensiva] [Tesis doctoral]. Universidad de Ottawa, Canadá.

Moreno, D., Estévez, E., Murgui, S. y Musitu, G. (2009). Relación entre el clima familiar y el clima escolar: el rol de la empatía, la actitud hacia la autoridad y la conducta violenta en la adolescencia. International Journal of Psychology and Psychological Therapy, 9(1), 123-136. http://www.uv.es/ lisis/david/psychological-therapy2.pdf

Pirani, Z., Faghihi, A. R. y Moradizade, S. (2016). Testing a Structural Equation Model Based on School Climate, Academic Self-efficacy and Perceived Social Support in High School Student in Lorestan Province, Iran [Probando un modelo de análisis estructural basado en clima escolar, autoeficacia académica y percepción de apoyo social en estudiantes de educación secundaria en Lorestan, provincial de Irán]. Turk Psikoloji Dergisi, 31(77), 81-88. https://bit.ly/2V7S86j

Riekie, H., Aldridge, J. y Afari, E. (2017). The role of the school climate in high school students' mental health and identity formation: A South Australian study [El papel del clima escolar en la salud mental y la formación de la identidad de los estudiantes de secundaria: un estudio de Australia del Sur]. British Educational Research Journal, 43(1), 95-123. https://doi.org/10.1002/berj.3254

Roffey, S. (2015). Becoming an agent of change for school and student well-being [Convertirse en un agente de cambio para el bienestar de la escuela y los estudiantes]. Educational and Child Psychology, 32(1), 21-30. http://www.su eroffey.com/wp-content/uploads/2014/02/2015-Becoming-an-Agent-of-Change-for-Wellbeing.pdf

Ruus, V-R., Veisson, M., Leino, M., Ots, L., Pallas, L., Sarv, E-S. y Veisson, A. (2007). Students' Wellk-Being, Coping, Academic Success, and School Climate [Bienestar, afrontamiento, éxito académico y clima escolar de los estudiantes]. Social Behavior and Personality, 35(7), 919-936. https://doi.org/10.2224/sbp.2007.35.7.919 
Ruvalcaba, N., Gallegos, J. y Fuerte, J. (2017). Competencias socioemocionales como predictoras de conductas prosociales y clima escolar positivo en adolescentes. Revista Interuniversitaria de Formación del Profesorado, 88(31.1), 77-90. https://www.redalyc.org/articulo.oa?id=27450136012

Smith, V.y Molina, M. (2011). Cuaderno metodológico 5. La entrevista cognitiva: guía para su aplicación en la evaluación y mejoramiento de instrumentos de papel y lápiz. Instituto de Investigaciones Psicológicas, Universidad de Costa Rica.

Turner, I., Reynolds, K., Lee, E., Subasic, E. y Bromhead, D. (2014). Well-Being, School Climate, and the Social Identity Process: A Latent Growth Model Study of Bullying Perpetration and Peer Victimization [Bienestar, clima escolar y procesos de identidad social: Un estudio de modelo de crecimiento latente sobre la perpetración del acoso y la victimización entre pares]. School Psychology Quarterly, 29(3), 320-335. https://doi.org/10.103 7/spq0000074

Urrelo, A. y Huamani, J. (2019). Satisfacción con la vida y estilos de afrontamiento en adolescentes de colegios públicos en la ciudad de Arequipa. Revista de Psicologia, 9(2), 13-32. https://revistas.ucsp.edu.pe/index.php/psicologia/ article/view/394

Villalobos, B., Carrasco, C., Olavarría, D., Ortiz, S., López, V., Oyarzún, D., Ascorra, P., Ayala, Á, Bilbao, Á., Morales, M. y Álvarez, J. (2016). Victimización de Pares y Satisfacción con la Vida: La Influencia del Apoyo de Profesores y Compañeros de la Escuela. PSYKHE, 25(2), 1-16. https://doi.org/10.7764/psykhe.25.2.861

\section{INFORMACIÓN ADICIONAL}

Cómo citar: Alvarado-Calderón, K. (2022). Exploración de las relaciones entre clima escolar, satisfacción con la vida y empatía en adolescentes costarricenses. Revista Educación, 46(1). http://doi.org/10.15517/revedu .$v 46 \mathrm{i} 1.45127$ 